\title{
IMPORTÂNCIA DE UM CURSO DE BOAS PRÁTICAS DE MANIPULAÇÃO PARA MANIPULADORES DE ALIMENTOS EM ESTABELECIMENTOS DE ALIMENTAÇÃO - ARTIGO DE REVISÃO
}

\author{
Fabio Taniwaki ${ }^{1}$ \\ Eduardo Delbon Baldini \\ Juliano Gonçalves Pereira \\ Rodrigo Iais da Silva \\ Marina Salvador Gonzalez Frontana \\ Carolina de Oliveira Arantes Jorge \\ José Rafael Modolo
}

\begin{abstract}
RESUMO
Anualmente, adoecem em média 600 milhões de pessoas por contaminação alimentar no mundo. As doenças transmitidas por alimentos (DTAs) representam um importante impacto à saúde pública, além de causarem prejuízo à economia do país por meio de custos diretos e indiretos. Devido ao contato direto, os manipuladores de alimentos (MA) favorecem a contaminação alimentar. Portanto, eles desempenham um papel importante na segurança e na preservação dos produtos alimentares. As boas práticas de manipulação de alimentos (BPMA) contribuem para a produção alimentar livre de microrganismos patogênicos. Por isso, torna-se essencial a qualificação técnica dos manipuladores, por meio de cursos de BPMA, a fim de garantir a qualidade higiênico-sanitária dos alimentos. No âmbito geral, é grande a necessidade de aprimorar a capacitação dos MA para que adquiram hábitos higiênico-sanitários adequados e melhorar a qualidade dos produtos e serviços. Com o objetivo de destacar a importância de um curso de capacitação de BPMA de estabelecimentos de alimentação, foi realizado um levantamento bibliográfico utilizando os Descritores em Ciências da Saúde, com as seguintes palavras-chave: boas práticas de manipulação, DTA, capacitação em boas práticas. Como o MA desempenha um papel importante para a saúde coletiva, espera-se que, com os temas sugeridos para o curso, o profissional possa implementar os ensinamentos de BPMA em suas atividades, contribuindo positivamente para a saúde pública e trazendo satisfação aos clientes de forma lucrativa para o empreendimento.
\end{abstract}

Palavras-chave: DTA, boas práticas, alimentos, manipuladores, saúde pública.

\section{IMPORTANCE OF GOOD HANDLING PRACTICES TRAINING FOR FOOD HANDLERS IN FOOD ESTABLISHMENTS}

\begin{abstract}
Every year, an average of 600 million people get sick from food contamination worldwide. Foodborne diseases (FBDs) have a major impact on public health and cause damage to the country's economy through direct and indirect costs. Due to direct contact, food handlers $(\mathrm{FH})$ favor food contamination. Therefore, they play an important role in the safety and preservation of food products. Good food handling practices (GFHP) contribute to food production free of pathogenic microorganisms. Thus, the technical qualification of the handlers is essential in order to guarantee the hygienic-sanitary quality of the food. In general, there is a great need to improve FH's training to acquire adequate hygienic-sanitary habits and to improve the quality

\footnotetext{
${ }^{1}$ Faculdade de Medicina Veterinária e Zootecnia - Câmpus de Botucatu. fahtani@ hotmail.com

Taniwaki F, Baldini ED, Pereira JG, Silva RI, Frontana MSG, Jorge COA, Modolo JR. Importância de um curso de boas práticas de manipulação para manipuladores de alimentos em estabelecimentos de alimentação - Artigo de revisão. Vet. e Zootec. 2020.; 27: 001-009.
} 
of products and services. In order to highlight the importance of a GFHP training course in food establishments, a bibliographic survey was conducted, using Health Sciences Descriptors, with the following keywords: good handling practices, FBD, good practices training. Since the FH plays an important role in public health, it is hoped that with the topics suggested for the course, they can implement the GFHP training in their activities, positively contributing to public health and improving customer satisfaction and profitability.

Keywords: FBD, good practices, food, handlers, public health.

\section{IMPORTANCIA DEL CURSO DE BUENAS PRÁCTICAS DE MANIPULACIÓN PARA MANIPULADORES DE ALIMENTOS EN ESTABLECIMIENTOS DE ALIMENTACIÓN}

\section{RESUMEN}

Cada año, un promedio de 600 millones de personas se enferman por contaminación de alimentos en el mundo. Las enfermedades transmitidas por los alimentos (ETA) tienen un gran impacto en la salud pública, además de dañar la economía del país a través de costos directos e indirectos. Debido al contacto directo, los manipuladores de alimentos (MA) favorecen la contaminación alimentaria. Por lo tanto, desempeñan un papel importante en la seguridad y conservación de los productos alimenticios. Las buenas prácticas de manipulación de alimentos (BPMA) contribuyen a la producción de alimentos libres de microorganismos patógenos. Por lo tanto, la calificación técnica de los manipuladores se vuelve esencial para garantizar la calidad higiénico sanitaria de los alimentos. En general, existe una gran necesidad de mejorar la capacidad de los MA para adquirir hábitos higiénicos y sanitarios adecuados y mejorar la calidad de los productos y servicios. Para resaltar la importancia de un curso de capacitación BPMA en establecimientos de alimentos, se realizó una encuesta bibliográfica, utilizando los Descriptores en Ciencias de la Salud, con las palabras clave: buenas prácticas de manipulación, ETA, capacitación en buenas prácticas. Dado que el manipulador desempeña un papel importante para la salud pública, se espera que con los temas sugeridos para el curso, el MA pueda implementar las enseñanzas de BPMA en sus actividades, contribuyendo positivamente a la salud pública y brindando satisfacción a los clientes de forma rentable a la empresa.

Palabras clave: ETA, buenas prácticas, alimentos, manipuladores, salud pública.

\section{INTRODUÇÃO}

As doenças transmitidas por alimentos e água (DTAs) são problemas de preocupação mundial. Morrem, no mundo, cerca de 420 mil pessoas por ano. Ainda, 550 milhões delas apresentam diarreia com 230 mil mortes anuais. O Brasil também merece atenção, de 2000 a 2015 foram registradas, no mínimo, 232 mil casos de DTAs e 115 mil mortes. Outras vítimas sofrem consequências como dores de cabeça, náuseas, vômitos, mal-estar (1).

Inquestionavelmente as DTAs causam consideráveis prejuízos diretos e indiretos aos órgãos públicos dos países (2).

A contaminação dos alimentos pode ser causada tanto pela manipulação inadequada no processo de industrialização quanto nos pontos de venda de destinação direta ao consumo humano, como restaurantes, quiosques, trailers e etc (3).

Enfatiza-se que, muitas vezes, essas atividades comerciais de venda de alimentação são iniciadas como uma fonte alternativa de renda para pessoas sem conhecimentos básicos em boas práticas de higiene, manipulação e conservação de alimentos (4). 
Portanto, os manipuladores podem ser uma das principais vias de contaminação dos alimentos e também desempenharem um papel importante na segurança higiênico-sanitária dos comestíveis em toda cadeia da produção até o consumidor final (5).

Entidades nacionais e internacionais de segurança dos alimentos reconhecem que a maioria das DTAs pode ser evitada a partir da manipulação adequada dos alimentos, quer na indústria, quer no comércio de venda direta ao consumo humano, e reconhecem ainda que se trata de um importante problema de saúde pública (6).

Assim, torna-se essencial para a prevenção das DTAs a qualificação técnica do pessoal que atua, sobretudo, na manipulação de alimentos preparados nos pontos de vendas de alimentação direto ao consumidor.

Ressalta-se que a capacitação em boas práticas é uma exigência legal para estabelecimentos comerciais de alimentos e serviços de alimentação destinados ao público (7).

Frente a esse cenário, os autores apresentam uma revisão da literatura para um curso com temas precisos e direcionados de capacitação em boas práticas de manipuladores de alimentos e que ela se destaca como um diferencial competitivo na qualidade da prestação de seus serviços.

\section{DESENVOLVIMENTO DO ASSUNTO}

Para cumprir com o ensinamento de capacitação básica em boas práticas, foi realizado um levantamento bibliográfico, utilizando os Descritores em Ciências da Saúde, com as seguintes palavras-chave: boas práticas de manipulação, DTA, capacitação em boas práticas. Os operadores booleanos utilizados foram "and" e "or". As bases de dados consultadas foram o Google Acadêmico, Scopus e BVS-VET.

Os critérios de inclusão utilizados foram: publicações dos últimos dez anos nas línguas portuguesa e inglesa, disponíveis na íntegra; relatos de casos; estudos qualitativos e quantitativos; cursos de manipulação de alimentos realizados.

Os critérios de exclusão utilizados foram: artigos em outras línguas; métodos que sugerissem viés científico; artigos não recuperados na íntegra.

Segundo a OMS, a promoção de saúde e manutenção da vida estão diretamente ligados ao acesso regular a alimentos seguros e nutritivos. No entanto, o consumo de alimentos contaminados pode causar mais de 200 doenças, desde diarreia até câncer (8).

Esta mesma entidade estima que a cada ano cerca de 600 milhões de pessoas adoecem no mundo, atingindo uma proporção de quase 1 em cada 10 pessoas, e 420.000 morrem por contaminação de origem alimentar. 40\% das DTAs atingem crianças menores de 5 anos, causando 125 mil mortes por ano. Além disso, as infecções diarreicas, que são mais comumente associadas ao consumo de alimentos contaminados, deixam 550 milhões de pessoas doentes todos os anos e causam 230.000 mortes (9).

No Brasil, dados apresentados pelo Ministério da Saúde mostram que, em 2017, foram notificados 598 surtos, com 9.320 doentes e 12 óbitos (10). Nesse mesmo período, as regiões Nordeste e Sudoeste tiveram o maior número de notificação de DTAs, seguidas das regiões Sul, Norte e Centro-Oeste. Os principais agentes etiológicos relacionados aos surtos foram Salmonella, Escherichia coli e Staphylococcus aureus (10).

Tal situação tem preocupado autoridades e órgãos privados vinculados aos problemas populacionais e alimentares devido à ligação direta com a produção de alimentos e a infraestrutura dessa população que cresce continuamente (6).

$\mathrm{O}$ aumento e a maior longevidade da população, o aumento do número de pessoas nos grupos de risco (idosos, gestantes, crianças e imunocomprometidos), o desordenado processo de urbanização, a globalização e a crescente necessidade de produção de alimentos em larga escala são fatores que contribuem para a maior ocorrência das DTAs (11). 
Outro fator que tem se destacado é o aumento da frequência da alimentação fora do domicílio. Devido a mudanças ocorridas nos hábitos alimentares da população, principalmente a correria do dia a dia, a alimentação fora de casa tornou-se uma alternativa conveniente e prática de alimentação que vem aumentando rapidamente. Esse crescimento resultou da maior variedade de serviços oferecidos, a melhora da qualidade desses serviços e a maior disposição das pessoas para gastos com alimentação e segurança alimentar (12).

Segundo a Pesquisa de Orçamento Familiar realizada de 2008 a 2009, 31\% das despesas das famílias com alimentos são destinadas à alimentação fora de casa (13).

Para corresponder a essa demanda tem aumentado a quantidade de diferentes pontos de comércio de alimentação, como restaurantes, quiosques, trailers etc.

Muitas vezes, essa atividade é iniciada como uma fonte alternativa de renda para pessoas sem conhecimentos básicos em boas práticas de higiene, manipulação e conservação de alimentos. Paralelamente ao aumento desse tipo de prestação de serviço, a ocorrência das doenças transmitidas por alimentos e água (DTAs) também tem aumentado (14).

No Brasil, do total de 6.809 casos de DTAs, aproximadamente 2.533 tem origem nas residências, 1.089 nos restaurantes, padarias ou similares e os demais casos são originados de outros locais tais como creches, escolas, eventos, entre outros (10). As condições impróprias do local de preparo e falta de conhecimento de técnicas de manipulação higiênico-sanitária por parte dos comerciantes e MA podem facilmente contaminar os alimentos, que se forem comercializados podem constituir um risco à saúde da população consumidora (15).

Não há garantia de inocuidade nos alimentos preparados fora de casa, pois, mesmo feitos no momento do pedido, os produtos ficam expostos ao ambiente e podem ser armazenados em recipientes e locais impróprios (16). O preparo antecipado, a grande quantidade e o inadequado processo térmico de armazenamento dos alimentos também podem levar à ocorrência de DTAs (14).

Todas as pessoas que participam dos processos de produção de um alimento, desde a fonte até o consumidor final, são consideradas MA. Devido ao contato direto com os alimentos, elas oferecem diversos meios de contaminação - mãos, ferimentos, boca, unhas, entre outros representando, assim, o principal veículo de contaminações em serviços de alimentação (17).

Portanto, a manipulação dos alimentos é uma das principais causas de contaminação alimentar, pois, se a higienização das mãos dos manipuladores, dos utensílios e das bancadas não for adequada, o alimento poderá ser contaminado (18).

A legislação vigente no estado de São Paulo é a Portaria no 5, de 9 de abril de 2013, do Centro de Vigilância Sanitária do estado de São Paulo, a qual aprova o regulamento técnico sobre boas práticas para estabelecimentos comerciais de alimentos e para serviços de alimentação. Ainda, define boas práticas como "procedimentos que devem ser adotados para garantir a qualidade higiênico-sanitária dos alimentos" (19).

No Capítulo II, Seção III, Artigo 17, da Portaria CVS 5/13, consta que, nos estabelecimentos comerciais de alimentos e serviços de alimentação, aos quais não se exige um responsável técnico profissional, a responsabilidade pela elaboração, implantação e manutenção de boas práticas pode estar a cargo do proprietário do estabelecimento ou de um funcionário capacitado, que trabalhe efetivamente no local, acompanhe integralmente o processo de produção e implemente os parâmetros e critérios estabelecidos nesse regulamento (19).

Além dessas determinações, esse funcionário deve ser comprovadamente submetido a um curso de capacitação em boas práticas oferecido por uma instituição de ensino ou qualificação profissional ou, ainda, pela vigilância sanitária, envolvendo os Procedimentos Operacionais Padronizados para higienização das instalações e do ambiente (19).

Cunha et al. (20), relataram que a gestão na qualidade de alimentos é auxiliada por diversas legislações a fim de oferecer alimentos seguros, além de favorecer a manutenção das 
empresas no mercado consumidor. Nos estudos que vem sendo realizados no país em relação à implantação de sistemas de gestão da qualidade em alimentos tem se destacado as Boas Práticas de Fabricação, o desconhecimento da legislação, a necessidade de capacitação específica para os manipuladores de alimentos visando, entre outros aspectos, fornecer subsídios para que eles assimilem os perigos associados à manipulação incorreta dos alimentos e os riscos gerados para o consumidor. Por isso, é de fundamental importância que os estabelecimentos do ramo possuam gestores e manipuladores de alimentos com conhecimentos e práticas de trabalho compatíveis às legislações vigentes e requisitos para segurança dos alimentos (20).

Segundo Winter et al. (21), a capacitação contribui para o aperfeiçoamento das técnicas e da manipulação adequada dos alimentos, além da efetiva mudança do comportamento e conscientização dos manipuladores.

Assim como Winter et al. (21), Cunha et al. (20) concluíram que as mudanças de comportamento dos manipuladores frente a seu trabalho foram resultantes de treinamento. Este deve ser um processo contínuo para que os manipuladores se conscientizem e assimilem a importâncias das suas ações. Além disso, o conteúdo da capacitação e a linguagem utilizada deve ser de fácil entendimento e o material informativo fornecido é importante para a compreensão do conteúdo abordado. Essas ações são imprescindíveis para que as Boas Práticas sejam implantadas e executadas de maneira eficaz, para assegurar a manutenção da qualidade do alimento (21).

Bastos et al. (22) enfatizaram que os treinamentos dos manipuladores devem ser periódicos, para sanar as dúvidas e assim contribuir para reduzir os riscos de contaminação e melhorar a segurança dos alimentos oferecidos.

Além de confirmarem que o curso de BPM proporcionou melhorias na qualidade higiênico-sanitárias dos estabelecimentos de serviço de alimentação, Sangioni et al. (23) concordaram que a educação dos manipuladores de alimentos por meio de cursos especializados deve ser continuada, incentivada e periodicamente ofertada com a fim de minimizar os agravos por DTAs à saúde pública.

As capacitações contribuem para que os procedimentos de higiene e manipulação dos alimentos sejam adequados. Por isso, considera-se fundamental a implantação das Boas Práticas visando a consolidação dos procedimentos necessários para a produção de um alimento seguro sob o ponto de vista higiênico-sanitário (24).

Devido a direta relação entre alimentos seguros, nutrição e segurança alimentar, os alimentos contaminados geram um ciclo vicioso de doenças e desnutrição, que afeta especialmente gestantes, crianças pequenas, idosos e doentes (9).

Melhorar a segurança alimentar é, segundo a FAO (25), a chave para alcançar os objetivos de desenvolvimento sustentável. Assim, os governos devem priorizar a segurança alimentar como sendo essencial para a saúde pública, uma vez que desempenham um papel fundamental no desenvolvimento de políticas e marcos regulatórios. Cabe a eles estabelecer e implementar sistemas efetivos de segurança alimentar que assegurem que produtores e fornecedores de alimentos ao longo de toda a cadeia alimentar operem de forma responsável e forneçam alimentos seguros aos consumidores (25).

\section{CONSIDERAÇÕES FINAIS}

Capacitar em boas práticas manipuladores de alimentos, é um fator determinante para reduzir os riscos de contaminação e melhorar a segurança higiênico-sanitária da alimentação oferecida ao consumidor contribuindo, assim, para prevenir as DTAs.

Portanto, como modelo da operacionalização do sistema de capacitação, são sugeridos os seguintes temas com suas ementas. A maioria deles em conformidade com a Portaria 5/2013

Taniwaki F, Baldini ED, Pereira JG, Silva RI, Frontana MSG, Jorge COA, Modolo JR. Importância de um curso de boas práticas de manipulação para manipuladores de alimentos em estabelecimentos de alimentação - Artigo de revisão. Vet. e Zootec. 2020.; 27: 001-009. 
do Centro de Vigilância Sanitária do estado de São Paulo, que regulamenta os serviços de alimentação no Estado.

\section{TEMA 1: BOAS PRÁTICAS COM COMPORTAMENTO DE EMPREENDEDORISMO}

Ementa: informar que o consumidor está cada vez mais atento e exigente para consumir alimentos seguros e que o diferencial de empreendedorismo na qualidade da prestação de serviço poderá proporcionar satisfação aos clientes de forma lucrativa para o empreendimento.

\section{TEMA 2: IMPORTÂNCIA DAS BOAS PRÁTICAS PARA A SAÚDE PÚBLICA}

Ementa: conscientizar sobre as doenças de origem alimentar, que constituem um grande e perigoso problema de saúde pública, e sobre a importância do papel do manipulador de alimentos, que é um ator insubstituível na prevenção da contaminação e transmissão dessas doenças, contribuindo para que os consumidores possam receber alimentos saudáveis.

\section{TEMA 3: MICRORGANISMOS}

Ementa: discorrer sobre as ações dos principais dos microrganismos patogênicos causadores das DTAs para os manipuladores possam compreender os reais motivos de adotarem os procedimentos preventivos dessas doenças

\section{OCORRE \\ TEMA 4: CONTAMINAÇÃO DE ALIMENTOS: COMO, ONDE E POR QUE ELA}

Ementa: apresentar os mecanismos de transmissão e contaminação dos agentes físicos, químicos e microbiológicos, bem como as formas de impedir a transmissão e contaminação dos alimentos.

\section{TEMA 5: HIGIENE DO MANIPULADOR}

Ementa: instruir a executar suas operações rotineiras pessoais de higiene para garantir aos clientes segurança, qualidade e confiança na alimentação oferecida.

\section{TEMA 6: HIGIENE DO LOCAL DE TRABALHO}

Ementa: instruir a executar suas operações rotineiras de limpeza e higiene do local de trabalho de forma adequada e rotineira, para garantir aos clientes qualidade e confiança no alimento oferecido.

\section{TEMA 7: ACONDICIONAMENTO E REFRIGERAÇÃO DOS ALIMENTOS}

Ementa: falar dos dois processos de acondicionamento e refrigeração como pontos fundamentais e críticos para a manutenção da segurança e das propriedades dos alimentos não perecíveis e perecíveis acondicionados.

\section{TEMA 8: ÁGUA TRATADA E A LIMPEZA DOS RESERVATÓRIOS}

Ementa: expor sobre a importância de consumir água tratada e sobre o correto acondicionamento e limpeza dos reservatórios para evitar a contaminação dos alimentos e a transmissão de doenças à população.

TEMA 9: ACONDICIONAMENTO, DESTINO ADEQUADO DO LIXO E CONTROLE DE PRAGAS URBANAS

Ementa: abordar sobre os cuidados adequados com o lixo e sua destinação correta, que representam uma questão de cidadania e de responsabilidade social, pois geram efeitos 
positivos para todos, como a proteção e manutenção da saúde humana e do meio ambiente, e, além disso, causam um impacto positivo na imagem da cidade.

É esperado que os temas propostos com seus objetivos definidos a uma capacitação básica em boas práticas, contribuam para que os manipuladores incorporem os ensinamentos sugeridos em suas atividades, acarretando satisfação e segurança higiênico-sanitária da alimentação oferecidas aos clientes, tendo uma contribuição positiva para a saúde pública.

E ainda, que essa atividade comercial é um diferencial competitivo na qualidade da prestação de seus serviços, gerando lucros para o empreendedor deste segmento de alimentação.

\section{REFERÊNCIAS}

1. World Health Organization - WHO. Food safety [Internet]. Geneva: WHO; 2019 [cited 2019 Jun 6]. Available from: www.who.int/news-room/fact-sheets/detail/food-safety

2. Grupo de Extensão para Segurança do Alimento - GESEA. Alimento seguro: o que significa? [Internet]. Piracicaba: Esalq; 2009 [2017 Jul 11]. Available from: http://www.esalq.usp.br

3. Lanza J. Surtos alimentares no Brasil [Internet]. FoodSafetyBrazil; 2016 [cited 2017 Jun 14]. Available from: http://foodsafetybrazil.org

4. Carneiro ACLL, Cardoso LM, Souza LT, Santos LV, Viana Filho GP. Elaboração de roteiro para inspeção das boas práticas de manipulação e comercialização de alimentos no setor informal. Vigil Sanit Debate. 2017;5(1):127-32. doi: http://dx.doi.org/10.22239/2317269x.00866.

5. Souza PA, Santos DA. Microbiological risk factors associated with food handlers in elementary schools from Brazil. J Food Saf. 2009;29(3):424-9. doi: 10.1111/j.17454565.2009.00166.x.

6. Silva JAB, Fontana RLM, Costa SS, Rodrigues AJ. Teorias demográficas e o crescimento populacional no mundo. Cad Grad Cienc Hum Soc Unit [Internet]. 2015 [cited 2019 Ago 7];2(3):113-24. Available https://periodicos.set.edu.br/index.php/cadernohumanas/article/viewFile/1951/1209

7. Secretaria da Saúde do Estado de São Paulo. Portaria CVS 5, de 9 de Abril de 2013. Aprova o regulamento técnico sobre boas práticas para estabelecimentos comerciais de alimentos e para serviços de alimentação, e o roteiro de inspeção, anexo [Internet]. São Paulo: Secretaria de Estado da Saúde; 2013 [cited 2019 Ago 14]. Available from: http://www.cvs.saude.sp.gov.br/up/PORTARIA\%20CVS-5_090413.pdf

8. Organização Pan-Americana da Saúde. Organização Mundial da Saúde Brasil. Segurança dos alimentos é responsabilidade de todos [Internet]. Brasília: OPAS, OMS; 2019 [cited 2019 Dec 18]. Available from: https://www.paho.org/bra/index.php?option=com_content\&view=article\&id=5960: seguran ca-dos-alimentos-e-responsabilidade-de-todos $\&$ Itemid $=875$ 
9. Organización Mundial de la Salud. Inocuidad de los alimentos [Internet]. Washington: OMS; 2019 [cited 2019 Dec 18]. Available from: https://www.who.int/es/news-room/factsheets/detail/food-safety

10. Brasil. Ministério da Saúde. Secretaria de Vigilância em Saúde. Departamento de Vigilância das Doenças Transmissíveis. Coordenação Geral de Doenças Transmissíveis. Surtos de doenças transmisíveis por alimentos no Brasil [Internet]. Brasília: Ministério da Saúde; 2018 [cited 2019 Aug 8]. Available from: https://portalarquivos2.saude.gov.br/images/pdf/2018/janeiro/17/Apresentacao-SurtosDTA-2018.pdf

11. Brasil. Ministério da saúde. Manual integrado de prevenção e controle de doenças transmitidas por alimentosn [Internet]. Brasília: Ministério da saúde; 2019 [cited 2019 Dez $18]$. Available from: http://bvsms.saude.gov.br/bvs/publicacoes/manual_integrado_prevencao_doencas_alime ntos.pdf

12. Leal D. Crescimento da alimentação fora do domicílio. Segur Aliment Nutr [Internet]. 2015 [cited 2019 Ago 10];17(1):123. Available from: https://periodicos.sbu.unicamp.br/ojs/index.php/san/article/view/8634806

13. Instituto Brasileiro de Geografia e Estatística - IBGE. IPCA varia 0,15\% em dezembro e fecha 2018 em 3,75\% [Internet]. Rio de Janeiro: IBGE; 2018 [cited 2019 Jun 7]. Available from: https://agenciadenoticias.ibge.gov.br/agencia-sala-de-imprensa/2013-agencia-denoticias/releases/23558-ipca-varia-0-15-em-dezembro-e-fecha-2018-em-3-75

14. Filgueiras BGA, Paula AH, Barbosa WM, São José JFB, Silva EMM, São José JFB, et al. Avaliação das Boas Práticas de Manipulação no fluxograma operacional de preparações cárneas servidas em uma unidade de alimentação e nutrição. Rev Inst Adolfo Lutz [Internet]. 2015 [cited 2019 Ago 9];74(2):162-8. Available from: http://www.ial.sp.gov.br/resources/insituto-adolfo-

lutz/publicacoes/rial/10/rial74_2_completa/rial74_2_completa/rial742.pdf

15. Carneiro ACLL, Cardoso LM, Souza LT, Santos LV, Viana Filho GP. Elaboração de roteiro para inspeção das boas práticas de manipulação e comercialização de alimentos no setor informal. Vigil Sanit Debate. 2017;5(1):127-32. doi: http://dx.doi.org/10.22239/2317-269x.00866.

16. Monteiro MAM. Caracterização do comércio ambulante de alimentos em Belo HorizonteMG. Demetra [Internet]. 2015 [cited 2019 Jun 7];10(1):87-97. Available from: https://www.e-publicacoes.uerj.br/index.php/demetra/article/view/13364

17. Ghisleni DR, Basso C. Educação em saúde a manipuladores de duas unidades de alimentação e nutrição do município de Santa Maria/RS. Discip Sci Ser Cienc Saude [Internet]. 2016 [cited 2019 Ago 7];8(1):101-8. Available from: https://periodicos.ufn.edu.br/index.php/disciplinarumS/article/view/905

18. Ponath FS, Valiatti TB, Sobral FDOS, Romão NF, Alves GMC, Passoni GP. Avaliação da higienização das mãos de manipuladores de alimentos do Município de Ji-Paraná, Estado de Rondônia, Brasil. Rev Pan-Amazonica Saude [Internet]. 2016 [cited 2019 Jun 
7];7(1):63-9.

Available

from:

http://scielo.iec.gov.br/scielo.php?script=sci_arttext\&pid=S2176-62232016000100008

19. Secretaria da Saúde do Estado de São Paulo. Portaria CVS 5, de 9 de Abril de 2013. Aprova o regulamento técnico sobre boas práticas para estabelecimentos comerciais de alimentos e para serviços de alimentação, e o roteiro de inspeção, anexo. São Paulo: Secretaria de Estado da Saúde; 2013 [cited 2019 Ago 14]. Available from: http://www.cvs.saude.sp.gov.br/up/PORTARIA\%20CVS-5_090413.pdf

20. Cunha FMF, Magalhães MBH, Bonnas DS. Desafios da gestão da segurança dos alimentos em unidades de alimentação e nutrição no Brasil : uma revisão. Contextos Aliment Rev Comport Cult Soc [Internet]. 2012 [cited 2019 Ago 9];1:4-14. Available from: http://www3.sp.senac.br/hotsites/blogs/revistacontextos/wpcontent/uploads/2013/04/Revista_Vol1_N24a14.pdf

21. Winter C, Medeiros LB, Serafim AL, Stangarlin-Fiori L. Avaliação da implementação das boas práticas de manipulação em unidade de acolhimento institucional por meio de um Programa de Incentivo fiscal e capacitação. Rev Inst Adolfo Lutz. 2015;74(1):75-80.

22. Bastos TB, Alves JDCL, Murino GB, Lenz FN. Curso de capacitação em Boas Práticas na manipulação de alimentos. Rev Eletronica Extensao. 2014;11(18).

23. Sangioni LA, Cadore GC, Botton AS, Vogel FSF, Silva ERA, Smaniotto H, et al. Impactos do curso de boas práticas de manipulação de alimentos em estabelecimentos de serviços de alimentação de Santa Maria, Rio Grande do Sul. Vet Zootec. 2019;26:1-8.

24. Brandão TBC, Silva MBB, Chicuta RL, Severiano GML, Silva EP. Evaluation of good practices in a food and nutrition unit of a municipal center for children education in Maceió, Alagoas. Hig Aliment [Internet]. 2019 [cited 2019 Dez 18];33:220-4. Available from: https://www.higienealimentar.com.br/wp-content/uploads/2019/06/Anais-Higienistas2019_web-2.pdf

25. Food and Agriculture Organization of the United Nations. World Health Organization. ICN2 Second International Conference on Nutrition: better nutrition better lives [Internet]. Rome: FAO, WHO; 2014 [cited 2017 Jun 14]. Available from: http://www.fao.org/icn2

\section{Recebido em: 05/09/2019 Aceito em: 13/05/2020}

